\title{
Un humaniste kierkegaardien à la recherche du sacré. Denis de Rougemont et le Collège de sociologie
}

\section{Alain Corbellari}

\section{CpenEdition}

Journals

Édition électronique

URL : https://journals.openedition.org/edl/1853

DOI : $10.4000 /$ edl. 1853

ISSN : 2296-5084

Éditeur

Université de Lausanne

\section{Édition imprimée}

Date de publication : 15 décembre 2019

Pagination : 71-84

ISBN : 978-2-940331-72-7

ISSN : 0014-2026

\section{Référence électronique}

Alain Corbellari, « Un humaniste kierkegaardien à la recherche du sacré. Denis de Rougemont et le Collège de sociologie », Études de lettres [En ligne], 311 | 2019, mis en ligne le 15 décembre 2021, consulté le 16 décembre 2021. URL : http://journals.openedition.org/edl/1853 ; DOI : https://doi.org/ 10.4000/edl. 1853

Ce document a été généré automatiquement le 16 décembre 2021.

(c) Études de lettres 


\title{
Un humaniste kierkegaardien à la recherche du sacré. Denis de Rougemont et le Collège de sociologie
}

\author{
Alain Corbellari
}

1 «Ce que j'éprouve maintenant, c'est cela qu'on doit appeler l'horreur sacrée $»^{1}$. Cette phrase célèbre du Journal d'Allemagne de Denis de Rougemont, écrite à propos du meeting nazi auquel il avait assisté le 11 mars 1936, et dont la description constitue ce que Denis Hollier appelle à juste titre le " morceau de bravoure $»^{2}$ du livre, a fait couler beaucoup d'encre ; surtout depuis que Bernard-Henri Lévy l'a utilisée avec une insigne mauvaise foi pour classer le grand apôtre du fédéralisme ${ }^{3}$ européen du côté des ennemis de la démocratie, n'hésitant pas à mettre en parallèle la fascination des fascistes français pour les jeunesses hitlériennes et «l'émoi de Denis de Rougemont devant ces "jeunesses bottées nu-tête, chemise ouverte, dont notre presse, dit-il, aime à railler les uniformes $" »^{4}$.

2 Est-il nécessaire de souligner ici que le Journal d'Allemagne inspira le Rhinocéros de Ionesco ${ }^{5}$ et constitue l'un des réquisitoires les plus lucides écrits contre le régime nazi avant l'éclatement de la Seconde Guerre mondiale ? ${ }^{6}$ Même si le ralliement fidéiste de Mounier à certains aspects de la politique de rénovation vichyste a lourdement grevé la crédibilité du personnalisme ${ }^{7}$, il est totalement illégitime d'associer Rougemont à cette dérive ${ }^{8}$.

3 Si cette évocation de l'«horreur sacrée " étonne sous la plume d'un penseur comme Denis de Rougemont, ce n'est donc pas parce qu'elle témoignerait d'un emballement suspect, mais plutôt parce qu'elle dévoile chez lui un penchant résolu pour les problématiques chères au Collège de sociologie. Porte-parole jusque là d'une forme d'existentialisme protestant d'obédience kierkegaardienne ${ }^{9}$ et théoricien d'une certaine idée de l'engagement de l'intellectuel (qui allait beaucoup inspirer, à partir de la Guerre, un certain Jean-Paul Sartre $)^{10}$, Rougemont était certes déjà connu comme 
passeur de la civilisation d'outre-Rhin (voire d'outre-Danube...) ${ }^{11}$, et on comprend que, opposant la "personne » (responsable) à "l'individu » (narcissique) pour renvoyer dosà-dos l'égoïsme libéral et l'égalitarisme communiste, il ne pouvait considérer les mouvements des masses que comme l'expression d'une aliénation. Dans Penser avec les mains, publié l'année même du séjour en Allemagne, il écrit d'ailleurs :

Tous ceux-là participent de la démission permanente de la pensée, de son inactualité, de sa séparation, de sa servitude inhumaine, de sa bassesse distinguée, de sa révolte contre la condition qui nous est assignée, - créatrice; tous ceux-là fondent, ici et maintenant, et dans leur vie, l'État totalitaire ${ }^{12}$.

4 Pour Rougemont, la «trahison des clercs» réside, plus encore que dans l'embrigadement idéologique dénoncé par Benda, dans la complicité passive; plus exactement, les deux attitudes lui semblent en fin de compte équivalentes puisqu'elles témoignent toutes deux d'un refus de penser qui sépare l'homme de ce qu'il a de meilleur : sa capacité à s'abstraire de ses contingences ataviques pour s'élever au statut de la «personne », autonome et concernée.

5 Dès son retour d'Allemagne, Rougemont se plonge dans l'écriture de ce qui allait être son livre le plus célèbre, L'amour et l'Occident, qui est très significativement, avec son complément ultérieur Comme toi-même, le seul de ses ouvrages à proposer un travail de critique littéraire sur nos origines culturelles ${ }^{13}$; de surcroît, il s'appuie sur l'élucidation d'un mythe, celui de Tristan et Iseut, dont Rougemont est ainsi devenu - bien qu'il ne fût pas médiéviste de profession - un interprète incontournable, participant du même coup à l'engouement de toute une époque pour une problématique qui faisait déjà craindre à Sartre qu'il n'existât désormais « un mythe du mythe »"

6 Au delà des approximations qui l'ont souvent disqualifié d'entrée de jeu aux yeux des spécialistes ${ }^{15}$, Rougemont se livre dans L'amour et l'occident à une description si frappante de l'amour passion - («Tristan et Iseut ne s'aiment pas, ils l'ont dit et tout le confirme. Ce qu'ils aiment c'est l'amour, c'est le fait même d'aimer $\left.\aleph^{16}\right)$ - que le message pourtant explicite du livre, à savoir l'injonction à se détourner de cette vision mortifère de l'amour (cette obsession d'un " amour réciproque malheureux $»^{17}$ ) pour se tourner vers un amour constructif, responsable et conjugal, a généralement moins retenu les lecteurs que l'apologie de l'irrépressibilité de la pulsion amoureuse. Au vrai, convaincre de la nocivité de la passion avait tout de la mission impossible face à l'imminente libération sexuelle qu'allait connaitre l'Occident ${ }^{18}$.

7 Nul hasard, donc, à ce que Rougemont soit sollicité, au moment de l'écriture de son livre, pour faire une conférence au Collège de sociologie, puisqu'il est alors tout particulièrement en phase avec la question de l'irrationnel qui est au centre des préoccupations de Bataille et de ses amis. Même si, dans les années suivantes, durant son exil américain, Rougemont fréquentera assidûment Breton et les surréalistes en exil, on peut dire que les années 1938-1939 représentent dans sa vie et sa carrière la pointe extrême d'une tentation irrationaliste qui donnera sens, par son dépassement, à ses engagements futurs en faveur de la construction européenne et de l'écologie.

Le Journal d'Allemagne, qui illustre bien cette tentation, n'est pourtant pour rien dans la proposition faite à Rougemont de parler au Collège, puisqu'on en trouve la première trace avant la parution de l'ouvrage ${ }^{19}$ dans une lettre de Roger Caillois conservée à la Bibliothèque publique et universitaire de Neuchâtel :

Mon cher Rougemont,

Un simple mot pour vous demander si la date du 22 nov. vous conviendrait pour 
l'exposé que vous nous avez promis au Collège de Sociologie? Choisissez le sujet. Mais je vous avoue une préférence toute personnelle en faveur de vos réflexions sur l'amour, telles que vous les avez inaugurées dans Esprit. Il sera facile, je pense, de leur donner un tour sociologique. Confirmez-moi par retour, voulez-vous? à cause de l'impression du programme ${ }^{20}$.

Notons que le très abondant Fonds Rougemont de la Bibliothèque de Neuchâtel ne contient aucune lettre de Leiris ou de Bataille. On trouve par contre dix-huit lettres de Caillois qui témoignent d'une forte et durable amitié entre les deux hommes. La lettre que l'on vient de citer est la première du lot, les autres s'étendent au moins (six ne sont pas précisément datées) jusqu'en 1966. Caillois ne semble ainsi jamais passer en Suisse sans s'arrêter aux portes de Genève, à Ferney, où habitait Rougemont. Leurs relations pendant la Guerre sont particulièrement intéressantes : c'est ainsi Caillois qui a décidé Victoria Ocampo, dès 1939, à faire traduire en espagnol le Journal d'Allemagne ${ }^{21}$; et une lettre du 3 avril 1941, écrite de Buenos Aires, témoigne sans équivoque de l'engouement de Caillois pour l'œuvre de Rougemont:

Vous devriez m'envoyer tout ce que vous publiez ou écrivez. Je le lirais d'abord. Et peut-être pourrait-on le publier ici. Je ne parle pas de Sur qui vous est naturellement ouvert, mais de l'éventualité d'une maison d'édition française dont le projet est à l'étude depuis que lesgens ont réfléchi sur le prix de vente des livres de Maurois, Romains et Maritain.

Caillois revient à la charge le 10 avril, félicitant Rougemont de son activité aux ÉtatsUnis :

J'admire, j'envie vos projets: de collaborations, d'ouvrages, de revues. Cette Amérique du Nord n'est pas si [jésuite ?] que vous dites. Ici les projets ne manquent pas. Ils ne se réalisent presque jamais, voilà tout.

11 Le 10 mai, infligeant un heureux démenti au pessimisme de cette dernière appréciation, Caillois annoncera cependant à Rougemont sa décision de lancer Les Lettres françaises ${ }^{22}$, dont on sait le glorieux destin.

12 Le 18 mars 1945, Caillois dit à Rougemont son enthousiasme, "admirant tout d'une manière égale et nouvelle (ou renouvelée) ", pour La part du diable, ce petit livre brillant et étonnant où l'essayiste protestant semble prendre au sérieux l'existence du plus vieil ennemi de l'humanité.

13 Ajoutons enfin au témoignage de la correspondance, l'anecdote narrée par Caillois dans L'incertitude qui vient des rêves ${ }^{23}$ : Caillois y raconte une rencontre "à l'improviste», en juillet 1952, avec Rougemont, à Strasbourg, où tous deux devaient quelques jours plus tard participer au même congrès: le ton de familiarité de la conversation rapportée (qui porte sur la difficulté de savoir si tel ou tel souvenir se rapporte à la réalité ou à une impression onirique) est révélateur d'une amitié tout à fait complice.

14 La relation privilégiée entre Caillois et Rougemont montre donc bien dans quel sens il faut prendre la participation de l'auteur de L'amour et l'occident aux travaux du Collège de sociologie : tenté par l'irrationnel, Rougemont ne perd cependant jamais pied; c'est avec le plus ironique et le plus distancié des trois mousquetaires du Collège qu'il dialogue essentiellement, en qui il trouve un interlocuteur capable d'apprécier ses affirmations parfois paradoxales en sachant qu'elles ne sont qu'un moment dialectique de sa réflexion, un frère en humanisme qui peut être titillé par certains vertiges, mais ne perd jamais son sang-froid face au déchaînement des passions humaines. 

d'introducteur à Rougemont: Pierre Klossowski qui avait évoqué le 19 mai l'interprétation kierkegaardienne d'Antigone. Proche à la fois des personnalistes (il a écrit dans Esprit) et du Collège, Klossowski offre ainsi un témoignage supplémentaire de la proximité d'intérêts entre les existentialistes chrétiens et le groupe de Caillois, Leiris et Bataille.

Reprenons donc la chronologie des relations entre Rougemont et le Collège, pour peu qu'elle soit exactement reconstituable. Le 30 juin 1936, Rougemont quitte l'Allemagne pour retrouver le groupe personnaliste et devenir en 1937 rédacteur en chef des Nouveaux Cahiers, auxquels collaborent entre autres Simone Weil et Boris Souvarine. Il commence alors la rédaction de L'amour et l'occident tout en restant très attentif à l'actualité, ce dont témoignera précisément le chapitre lu au Collège de sociologie le mardi 29 novembre $1938^{24}$, en une séance qui ne suivra que de deux mois la signature des accords de Munich à laquelle Rougemont avait par ailleurs réagi à chaud en publiant dans la Nouvelle revue française un bref et incisif commentaire intitulé «Page d'histoire ». C'est aux mêmes «événements de septembre " que réagira Bataille dans la conférence qui suivra celle de Rougemont le 13 décembre, la saison ayant été ouverte le 15 novembre par Caillois qui y évoquait «L'ambiguïté du sacré ». La conférence de l'essayiste suisse ne pouvait être mieux mise en valeur. Que Caillois ait par ailleurs fait son miel du récit rougemontien du meeting nazi, ainsi que de l'article «Page d'histoire », on en veut pour preuve ces lignes écrites le 7 novembre :

Mon cher Rougemont,

Merci de votre Journal d'Allemagne. Je l'ai beaucoup aimé. Il n'est rien qui ne me paraisse juste dans vos observations et moralement, cela me semble d'une grande force. Je crois très important votre diptyque des discours Hitler-Niemüller.

Encore plus d'accord avec vos deux pages de la dernière N.R.F. C'est beaucoup plus «Collège de sociologie » que notre déclaration même : certains paragraphes disent ce que nous aurions dû dire. J'en suis un peu honteux. (Je fais surtout allusion à ce [que] vous dites du côté rituel de l'entrée en armes) [...].

De fait, «Page d'histoire » est, en dépit de sa brièveté, l'un des textes politiques les plus brillants de Rougemont: dénonçant l'hypocrisie des démocraties occidentales dont le « jacobinisme [...] (centralisation rigide, confusion de l'État et de la Nation)» contredit à la fois les buts de la S.D.N. et l'affirmation du droit des peuples à l'autodétermination, il raconte en sept points les accords de Munich comme l'aurait fait un manuel d'histoire du futur. Rougemont présente ainsi comme inéluctables des «événements ultérieurs (colonisation intra-européenne, état de guerre non déclarée) » dont il craint sans doute qu'ils soient - aussi « surprenants et monstrueux » qu'ils puissent apparaître - dépassés par la réalité.

Le passage auquel fait référence Caillois est, de toute évidence, l'analyse, évidemment marquée par l'expérience allemande de Rougemont, de l'idéologie hitlérienne :

Hitler démasqua l'aspect original (et non plus jacobin) de la dictature totalitaire: l'impérialisme religieux ou sacral. Il exigea d'entrer en armes et sur le champ dans les territoires Sudètes. Une cession purement diplomatique n'eût pas compté à ses yeux. La religion dont il était le fondateur voulait le sacrifice sanglant (ou son symbole), le viol de la victime, la «libération » violente de la proie désirée (guerre limitée) ${ }^{25}$.

On comprend qu'un membre du Collège de sociologie ait pu être jaloux de ces lignes qui allaient droit au but avec un aplomb qu'un Suisse pouvait sans doute davantage se 
permettre qu'un Français. Rougemont ne pouvait par ailleurs s'empêcher d'évoquer in fine le modèle qu'auraient dû adopter les démocraties occidentales : «l'utopie agissante d'une Fédération des Égaux, dont la Suisse figurait le microcosme». Après avoir renchéri, mais à titre de repoussoir, sur la conception chère à Bataille d'une violence du sacré, l'essayiste neuchâtelois aboutit à l'une des premières formulations de son credo euro-fédéraliste. Article charnière s'il en est, «Page d'histoire » ne marche donc sur les brisées du Collège de sociologie que pour mieux affirmer sa foi en un triomphe ultime de la raison.

On n'a retrouvé dans le Fonds Rougemont de la Bibliothèque de Neuchâtel aucune trace de la conférence du Collège de sociologie, signe que Rougemont a très vraisemblablement utilisé le texte manuscrit du livre en cours. Notons que le chapitre choisi, «Amour et guerre ", est celui-là même - le seul! - que Sartre, dans son compte rendu acerbe de L'amour et l'Occident, avouera trouver " excellent " ${ }^{26}$. C'est, de fait, l'un de ceux où Rougemont a le mieux senti l'air, particulièrement électrique, du temps. Sa proximité avec les idées de Caillois n'a jamais été aussi grande. On retrouve en effet chez les deux auteurs l'idée que la guerre est une pulsion fondamentale de l'humanité et qu'elle se manifeste à travers des substituts qui en détournent et en atténuent la violence brute. Si Caillois s'est longuement penché sur la sublimation de la violence guerrière dans le jeu ${ }^{27}$, il dressera dans Bellone, l'un de ses derniers ouvrages, un bref tableau de l'histoire de la guerre en Occident qui doit à l'évidence beaucoup au panorama proposé dans L'amour et l'occident. Le but avoué de Rougemont, est d'analyser les liens entre (les termes sont importants) "l'instinct de guerre et l'érotisme ${ }^{28}$ ", autrement dit entre la pulsion de mort et le principe de plaisir, même si Rougemont réfute explicitement la référence à Freud :

Il n'est pas nécessaire par exemple de recourir aux théories de Freud [...] : les

figures courantes du langage le font voir avec plus d'évidence ${ }^{29}$.

21 À une méthode herméneutique qui dégagerait l'impensé de notre rapport à la guerre, Rougemont préfère donc une analyse descriptive qui parcourra l'entier du champ historique occidental du Moyen Âge à nos jours. Le chapitre fait la transition entre celui consacré aux avatars littéraires du mythe de Tristan et ceux, conclusifs (et généralement oubliés des lecteurs), où s'affirme la nécessité du rejet du mirage tristanien et d'une revalorisation de l'institution du mariage. C'est dire l'enjeu rhétorique de la démonstration sur la guerre dans l'économie de l'ouvrage !

Les analyses de Rougemont sont brillantes et pleines de trouvailles: ainsi de la comparaison entre la séduction de ses deux épouses par Napoléon et l'évolution de ses conceptions stratégiques. On se perdrait à tout citer; bornons-nous à constater que le parallèle amour-guerre ne se laisse jamais oublier; aisé lorsqu'il s'agit de comprendre les conceptions courtoises du Moyen Âge au travers de la formule canonique arma et amor ou lorsque la "guerre en dentelles» est mise en rapport avec le libertinage du siècle de Louis $\mathrm{XV}$, il devient plus ardu lorsqu'on aborde le $\mathrm{XX}^{\mathrm{e}}$ siècle :

À partir de Verdun, que les Allemands appellent la Bataille du matériel (Materialschlacht), il semble que le parallélisme institué par la chevalerie entre les formes de l'amour et de la guerre, soit rompu ${ }^{30}$.

23 D'où cette conclusion que Rougemont prétend avoir été «loin de prévoir en commençant ce livre $»^{31}$ : la guerre totale, comme l'excès de la passion, mène à la négation de ce qui lui donnait sens. Doit-on en conclure qu'une forme "policée » de guerre semblerait civilisationnellement viable à Rougemont? Le parallèle l'exigerait, 
mais Rougemont met en pièces cette fausse évidence en émettant la remarque curieuse que « dans le domaine de la guerre, [...] toute évolution est pratiquement irréversible alors qu'il y a des retours littéraires $\|^{32}$, comme si la technique de la guerre destructrice totale n'avait pas déjà été pratiquée par Assurbanipal, Attila, Basile II ou Gengis Khan, et comme si le Moyen Âge, précisément, n'avait pas tenté de civiliser par la littérature une pratique dont l'absurdité ne lui échappait pas totalement.

La conclusion de Rougemont se veut donc rassurante, mais elle peut apparaitre naïve, et les trois quarts de siècle qui nous séparent de son analyse nous en démontrent toute la fragilité: si réellement la peur d'une destruction totale a pu, dans une certaine mesure, dissuader l'Occident - mais seulement après un conflit qui était en 1939 encore à venir ! - de s'entre-déchirer, elle ne l'a certes pas empêché de lancer à sa périphérie de nouvelles guerres pour lesquelles on s'est bien accommodé de "revenir» à des formes de guerre un peu moins massivement destructrices que celle dont nous avons désormais les moyens.

Reste une question lancinante : si la civilisation ne peut que gagner à bannir la guerre, la force du parallèle n'incite-t-elle pas Rougemont à promouvoir un amour à ce point dépassionné qu'il en viendrait à rejoindre les sinistres visions de Brave New World? On ne lit évidemment rien de tel dans L'amour et l'occident, mais en sortant ce chapitre de son contexte pour le présenter au Collège de sociologie, Rougemont en a sans nul doute accentué la portée provocatrice. La mise en parallèle de l'exaltation de la guerre et de l'éloge de la passion avait en effet tout pour enthousiasmer un public chauffé à blanc par les récents événements politiques, et, sous le couvert d'un discours raisonnable et sagement argumenté, on peut gager que les auditeurs du 29 novembre n'ont pas manqué de ressentir, même fugitivement, un frisson « d'horreur sacrée ».

\section{BIBLIOGRAPHIE}

ACKERMANN, Bruno, Denis de Rougemont : une biographie intellectuelle, Genève, Labor et Fides, 1996, 2 vols.

-, Denis de Rougemont. De la personne à l'Europe, préface de Henri-Charles Tauxe, postface de Claude Haegi, L'Âge d'homme, Lausanne, 2000.

BEAUVoIR, Simone de, Mémoires, 2 vols, Paris, Gallimard, 2018, (« Bibliothèque de la Pléiade »).

CAILloIs, Roger, L'homme et le sacré, Paris, Gallimard, « Folio essais », 1950.

-, L'incertitude qui vient des rêves, Paris, Gallimard, 1956.

-, Bellone ou la pente de la guerre, Saint-Clément-la-Rivière, Fata Morgana, 1994.

CORBELLARI, Alain, Prismes de l'amour courtois, Dijon, Éditions universitaires de Dijon, 2018.

HOLLIER, Denis, Le Collège de sociologie 1937-1939, Paris, Gallimard, « Folio Essais », 1995.

LÉVY, Bernard-Henri, L'idéologie française, Paris, Grasset, 1981.

Études de lettres, $311 \mid 2019$ 
PERISANU, Mariana, «Le nazisme et le communisme : peurs et antidotes dans les écrits d'Ionesco », Travaux de littérature, 17 (2004), p. 479-491.

ROUGEMONT, Denis de, «La Jeunesse française devant l'Allemagne (Réponse à l'enquête) », La Revue du siècle, 2 (mai 1933), p. 7-9.

-, Penser avec les mains, Paris, Albin Michel, 1936.

_, Journal d'Allemagne, Paris, Gallimard, 1938 ; rééd. in Journal d'une époque, Paris, Gallimard, 1968

-, «Page d'histoire », Nouvelle Revue Française, 51 (1938/2), «L'Air du mois », p. 866-867.

-, L'amour et l'Occident, Paris, « 10/18 », 1992 (1939).

_, L'aventure occidentale de l'homme, Paris, Albin Michel, 1957.

-, Comme toi-même, Paris, Albin Michel, 1961, rééd. sous le titre Les mythes de l'amour, Paris, Gallimard, 1972.

-, Vingt-huit siècles d'Europe, Paris, Payot, 1961.

-, La Suisse ou l'histoire d'un peuple heureux, Paris, Hachette, 1965.

SARTRE, Jean-Paul, « La Chronique de Jean-Paul Sartre », Europe, 198 (15 juin 1939), p. 240-248 ;

rééd. in Situations I, Paris, Gallimard, 1947, p. 75-84.

STERNHELL, Zeev, Ni droite ni gauche. L'idéologie fasciste en France, Paris, Éditions du Seuil, 1983.

\section{NOTES}

1. D. de Rougemont, Journal d'une époque, p. 320.

2. D. Hollier, Le Collège de sociologie 1937-1939, p. 406.

3. Pour nombre de politiciens français, aujourd'hui encore, «fédéralisme » n'est pas loin d'être un gros mot (rimant fâcheusement avec « féodalisme »).

4. B.-H. Lévy, L'idéologie française, p. 30. La citation de Rougemont est tirée de «La Jeunesse française devant l'Allemagne (Réponse à l'enquête) », p. 7.

5. M. Perisanu, dans «le nazisme et le communisme ", p. 485, ne fait pas le lien de manière précise mais cite toutefois Rougemont en bonne compagnie (Malraux, Camus, Bernard-Henri Lévy...) parmi les grands dénonciateurs du totalitarisme qui ont abondé dans le sens de Ionesco.

6. Pensons à la terrible clausule du compte rendu : «Je suis seul et ils sont tous ensemble » (D. de Rougemont, Journal d'une époque, p. 320).

7. Voir en particulier Z. Sternhell, Ni droite ni gauche, p. 299-310.

8. Voyant qu'un journaliste dans un compte rendu du livre de B.-H. Lévy avait fait le pas supplémentaire d'assimiler directement Rougemont à Brasillach et à Doriot cités conjointement, l'essayiste neuchâtelois intentera une plainte en justice et obtiendra gain de cause.

9. D. Hollier (Le Collège de sociologie, p. 256) a souligné à très juste titre l'importance du compte rendu des Propos sur la religion d'Alain, publié par Rougemont en 1939 dans la N.R.F., pour comprendre ce qui sépare un fidéiste attaché aux institutions religieuses comme Alain, d'un penseur pour qui la foi est et doit rester un scandale pour la raison.

10. On peut d'ailleurs soutenir que les raisons pour lesquelles ce dernier consacra à L'amour et l'Occident un compte rendu resté célèbre, republié dans Situations, sont liées au besoin qu'avait Sartre de discréditer l'un de ses principaux concurrents sur la scène intellectuelle française, manœuvre qui réussit d'autant mieux que Rougemont eut le tort stratégique de rester en 
Amérique durant toute l'Occupation, et qu'il ne put jamais reconquérir après la Guerre l'audience considérable qui avait été la sienne à la fin des années 30 .

11. Je songe bien sûr ici au Paysan du Danube, premier volume du Journal rougemontien.

12. D. de Rougemont, Penser avec les mains, p. 192.

13. Vingt-huit siècles d'Europe fait, à l'aide de morceaux choisis, l'histoire de l'idée d'Europe ; La Suisse ou l'histoire d'un peuple heureux présente l'histoire et les institutions de la Suisse à ceux qui ne la connaissent pas, L'aventure occidentale de l'homme propose une synthèse à la politique et culturelle. Mais aucun de ces ouvrages n'insiste proprement sur la littérature.

14. J.-P. Sartre, « La Chronique de Jean-Paul Sartre », p. 77.

15. Deux griefs peuvent, en particulier, être faits à l'ouvrage: l'affirmation d'un lien non démontré et intuitivement paradoxal entre l'ascétisme cathare et le gay savoir des troubadours, et l'assimilation abusive de l'amour passion égalitaire qui unit Tristan et Iseut à la pulsion fondamentalement inégalitaire (l'amant est l'esclave de sa dame) de la fin'amour. Je me permets de renvoyer pour plus de précisions à mon ouvrage Prismes de l'amour courtois, en part. p. 105 sq.

16. L'amour et l'Occident, p. 43.

17. Ibid., p. 55.

18. Significatif à cet égard est le petit scandale provoqué il y a quelques années par la divulgation de la liaison extra-conjugale que Rougemont avait entretenue avec l'épouse de... son ami SaintExupéry (les deux écrivains furent particulièrement proches à l'époque de l'écriture du Petit Prince; on raconte que c'est Rougemont lui-même qui prenait la pose lorsque Saint-Ex croquait les attitudes de son personnage ; voir B. Ackermann, Denis de Rougemont, p. 11 sq.).

19. Achevé d'imprimer le 12 octobre 1938. On lira plus loin la lettre écrite par Caillois le 7 novembre, témoignant de sa toute fraîche lecture du Journal d'Allemagne.

20. Lettre de Roger Caillois à Denis de Rougemont, le 15 août 1938. Bibliothèque publique et universitaire de Neuchâtel, Fonds Denis de Rougemont, ID 5107. Je remercie Jonathan Wenger de son aide dans la transcription des textes.

21. La traduction paraîtra en 1941.

22. B. Ackermann, dans sa bibliographie (Denis de Rougemont, p. 1183), recense trois contributions de Rougemont à Sur et une aux Lettres françaises en 1941.

23. R. Caillois, L'incertitude qui vient des rêves, p. 31 ss.

24. Et non le 22 comme primitivement proposé par Caillois.

25. D. de Rougemont «Page d'histoire», p. 866 sq.

26. J.-P. Sartre, Situations, I, p. 76. Pour Simone de Beauvoir, c'était plutôt lorsqu'il parlait de sexualité que Rougemont était à son meilleur. Elle évoque en effet dans La force des choses (in S. de Beauvoir, Mémoires, t. II, p. 185) «Rougemont qui parle sottement de l'Europe, mais pas si mal de sexe ».

27. Voir l'appendice « Guerre et sacré » dans R. Caillois, L'homme et le sacré, p. 219-242.

28. D. de Rougemont, L'amour et l'Occident, p. 265.

29. Ibid.

30. Ibid., p. 289.

31. Ibid., p. 295.

32. Ibid. 


\section{RÉSUMÉS}

Les relations entre Rougemont et le Collège de sociologie, par l'intermédiaire essentiellement de Roger Caillois, avec qui il gardera des liens assez étroits, se cristallisent autour d'une conférence donnée le 29 novembre 1938, dans le cadre du Collège, qui reprend le chapitre «Amour et guerre " de L'amour et l'Occident, alors en cours d'écriture. On comprend mieux, à relire ce texte, la notion d'« horreur sacrée » utilisée dans un moment crucial du Journal d'Allemagne.

\section{AUTEUR}

\section{ALAIN CORBELLARI}

Universités de Lausanne et de Neuchâtel 